\title{
La socialité du texte dans Une saison au Congo d'Aimé Césaire: Approche sociocritique
}

\author{
Manal Mamdouh Youssef Ahmed \\ Faculté de l'Al-Alsun, Université de Minia, Égypte \\ Email: manal.yousef@mu.edu.eg
}

The social character of the text in Une saison au Congo by Aimee Césaire: A critical approach of societal conditions

Although we live in Africa 'the black continent', we are ignorant of blacks. So, this play has been chosen for study as an attempt to offer the nature as well as culture of one of these societies

Poet and playwright Aimee Césaire (1913-2008) was concerned with issues of blacks, colonialism, and slavery. In the play Une saison au Congo (1973), Césaire dealt with the reign of Lumumba, who started liberating the Congo and wanted to accomplish this enormous task in a short time. But Lumumba was surrounded by domestic as well as foreign conspiracies. The play ends with the assassination of Lumumba and represents many political, social, and cultural aspects characterizing the Congo. It could be said that the play is a historical work as it offers historical events as they occurred though in a literary style.

In the current study, there is an attempt to answer the following research questions: How did Césaire depict the relationship between the colonizer and the colonized? How did the Congolese society develop following independence? What are the problems faced by the people of the Congo after independence? What are the political and social customs that Césaire criticized in the Congolese society?

This study is based on Claude Duchet's societal critical approach and is divided into three sections: political aspects, social aspects, and cultural aspects.

Keywords: decolonization, blacks, Lumumba, Claude Duchet, Congolese culture 


\section{Résumé}

Père de mouvement de la négritude, poète et dramaturge, Aimé Césaire (1913-2008) prend en charge la question de la négritude, de la colonisation et de l'esclavage. Dans Une saison au Congo (1973), Césaire expose la période du règne de Lumumba qui se lance à la conquête de la libération du Congo. Il veut réaliser cette tâche immense en un temps-record. Mais, il était entouré de complots à l'intérieur et à l'extérieur. La pièce se termine par l'assassinat de Lumumba.

Dans la présente étude nous cherchons à répondre aux questions suivantes: comment aborde-Césaire la relation entre le colonisateur et le colonisé ? Comment la société Congolaise a-t-elle évoluée après l'indépendance ? Quels sont les problèmes qu'affrontent les Congolais après l'indépendance ? Quelles sont les mœurs politiques et sociales critiquées de la part de Césaire?

La présente étude s'appuie sur la sociocritique de Claude Duchet. Elle se compose en trois axes : les aspects politiques, les aspects sociaux et les aspects culturels.

Mots Clés: décolonisation, nègre, Lumumba, Claude Duchet, culture congolaise

\section{Introduction}

Père du mouvement de la négritude, poète et dramaturge, Aimé Césaire $(1913,2008)$ prend en charge la question de la négritude, de la colonisation et de l'esclavage. Dans Une saison au Congo (1973), Césaire expose la période du règne de Lumumba qui se lance à la conquête de la libération du Congo. Il veut réaliser cette tâche immense en un temps record. Mais, il était entouré de complots à l'intérieur et à l'extérieur. La pièce se termine par l'assassinat de Lumumba.

Pour ne pas tomber dans le déjà dit, nous ne cherchons pas à quel point la pièce s'identifie au réel, car, dans son livre "Quand Césaire écrit Lumumba parle", Suzanne Houyoux montre que la pièce s'identifie bien au réel:

Aimé Césaire s'est informé de la pensée politique de Lumumba et en rend 
compte dans sa pièce; il mentionne des lieux comme des événements précis qui ont fait l'actualité politique du moment; ainsi, je suis portée à dire que Césaire écrit bien l'histoire. (Houyoux, 1993, p. 14).

Dans la présente étude nous cherchons à répondre aux questions suivantes : comment aborde-Césaire la relation entre le colonisateur et le colonisé ? Comment la société Congolaise a-t-elle évolué après l'indépendance ? Quels sont les problèmes qu'affrontent les Congolais après l'indépendance ? Quelles sont les mœurs politiques et sociales critiquées de la part de Césaire?

La présente étude s'appuie sur la sociocritique de Claude Duchet. Elle se compose de trois axes : les aspects politiques, les aspects sociaux et les aspects culturels.

\section{Préambule théorique}

Qu'est-ce que la sociocritique ? Quels sont les outils indispensables à l'analyse sociocritique d'un texte littéraire? La sociocritique se compose de deux éléments "socio-critique"1: le préfixe socio désigne la relation avec la société et le suffixe critique désigne la relation avec les textes et la littérature. Les deux pôles de la sociocritique sont : société - littérature. La relation entre la littérature et la société est très forte et bien connue. L'œuvre littéraire est une œuvre de communication, elle reflète les marques de la société. L'influence de la littérature sur la société est considérable; les œuvres littéraires peuvent sortir l'homme de son ignorance.

La sociocritique est une théorie littéraire dont l'initiateur est Claude Duchet et le cofondateur est Edmond Cros. La première formulation de la sociocritique $^{2}$ se trouve dans l'article "Pour une socio- critique ou variations sur un incipit “, paru dans la revue Littérature, février 1971.

\footnotetext{
${ }^{1}$ La "sociocritique" était initialement formulée avec un trait d'union, et s'écrivait ainsi: socio-critique pour mettre l'accent sur les deux termes: la société et la critique.

${ }^{2}$ On remarque pour une première fois une approche sociale de la littérature dans "l'Émile"de J.-J. Rousseau puis, de manière plus importante, dans l'ouvrage "De la littérature considérée dans ses rapports avec les institutions sociales" (1800) de Mme de Staël; elle tente de cerner l'influence de la littérature sur les institutions sociales, la religion, les mœurs, les lois et aussi son contraire, c'est-à-dire l'impact de la vie morale et politique sur l'esprit de la littérature.
} 
Avant de présenter la sociocritique et ses éléments, il faut noter que certains ont tendance à confondre la sociologie de la littérature et la sociocritique. Claude Duchet a montré, dans l'article mentionné audessus, que les deux disciplines sont différentes.

La sociologie de la littérature est: "une partie intégrante de la sociologie [qui] tenterait d'appliquer les méthodes de la sociologie à la diffusion, aux succès et au public, à l'institution littéraire, aux groupes professionnels tels qu'écrivains, professeurs ou critiques" (Leenhardt, 2004). Donc, la sociologie de la littérature s'intéresse à tout ce qui est dans le texte, pas le texte lui-même. Barberis indique que la sociologie du texte littéraire concerne l'amont (conditions de production de l'écrit) et que la sociologie de la réception et de la consommation concerne l'aval (lectures, diffusion, interprétations, destins culturels et scolaires ou autres) (Bergez, 1996). Selon Claude Duchet, la sociocritique vise "le texte lui-même comme lieu où se joue et s'effectue une certaine socialité” (Bergez, 1996, p. 123). La sociocritique est donc une approche du fait littéraire qui s'attarde sur l'univers social présent dans le texte.

La sociocritique s'inspire bien de la sociologie de la littérature. C'est la problématique qu'affronte la sociocritique dès ses débuts et au cours de ses développements, mais Claude Duchet n'a pas cessé d'éclairer la spécificité de sa méthode; dans son entretien avec Ruth Amossy en 2005, il annonce clairement que: "la sociocritique n'est pas une sociologie de la littérature" (Amossy, 2006, p. 136). La sociocritique s'interroge sur l'implicite, les présupposés, le non-dit ou l'impensé et les silences. Il souligne que le but de la sociocritique est d'analyser la socialité de texte:

La socialité du texte s'atteint par une lecture interne, immanente, textualiste pour reprendre des mots chargés de mépris par les sociologues: “C'est dans la spécificité esthétique même, la dimension valeur des textes, que la sociocritique s'efforce de lire cette présence des œuvres au monde qu'elle appelle la socialité". (Duchet, 1979, p. 4).

Le triptyque "information, indice, valeur "est pour Duchet les paliers méthodologiques de sa démarche:

Chaque élément du texte, un personnage, une heure, un lieu, une notion abstraite, existe dans le texte selon trois modalités : une information sur le monde, un signe d'autre chose que lui-même [...] et une valeur $[\ldots]$, chaque 
élément prend sa valeur par un système d'opposition avec les autres éléments du texte. (Galvez, 2014, p. 22)

Duchet a posé une question importante pour montrer la visée de la science des textes: "Que serait la science des textes si elle ne nous remettait en possession du monde, à travers le lire et la parole humaine ? Lire pour voir clair, lire pour apprendre et s'apprendre" (Duchet, 2011, p. 83). La réponse à cette question concrétise notre démarche dans cette étude; nous travaillons pour faire voir, pour faire apprendre tout ce qui concerne la société nègre (congolaise) à travers cette pièce.

Nous travaillons sur la société du texte, la société référence et le cotexte. La société du texte : c'est la société qui se dégage du texte littéraire. La société référence: c'est la société-mère, une société où l'auteur puise des faits sociaux qu'il transformera ensuite en fait littéraire. Le contexte est: "ce qui dans le texte ouvre à un dehors du texte, sur un ailleurs du texte, sur un domaine de référence avec lequel le texte travaille" (Duchet, 1983, p. 26). Duchet voit que le texte se nourrit de ses références, mais, tout en s'en nourrissant, provoque d'autres références. Le but essentiel de la sociocritique est: rechercher "la socialité "du texte. Cette socialité évoquée dans le texte crée une société qui s'exprime par des structures, un fonctionnement, une hiérarchie sociale, des valeurs, des idéologies, les modes de penser qui lui appartiennent en propre.

\section{Les aspects politiques}

Césaire, en commentant son théâtre, dit que son objectif est, au premier degré, politique. C'est l'aspect politique qui domine dans son théâtre, car il a déjà dit que les problèmes majeurs de l'Afrique sont politiques. Dans son livre "La Littérature Nègre" Chevalier voit que : "Le théâtre africain semble se développer dans trois directions principales : la dénonciation du colonialisme et de ses séquelles, l'analyse des conflits des générations et la critique de mœurs politiques" (Chevalier, 2004, p. 161). Notre pièce est classée sous la troisième direction.

La pièce jette la lumière sur trois séquences de l'histoire du Congo : quelques mois avant l'indépendance, les mesures de l'indépendance et après l'indépendance (la saison du règne de Lumumba). La séquence de l'avant l'indépendance est assez dense (elle forme les quatre premières 
scènes du premier acte), Césaire l'expose pour faire voir comment était le Congo avant l'indépendance. C'est une séquence assez importante, car elle donne au lecteur un arrière-plan sur la colonisation au Congo en révélant, loin de toutes retouches fictives, la relation entre le Nègre et le Blanc. Ce qui nous intéresse, c'est la scène du bonimenteur qui vend la bière. Le bonimenteur est la figure de Lumumba: "Pour présenter son personnage, Césaire a puisé dans la réalité car Patrice Lumumba était effectivement directeur commercial de la marque de bière Polar en 1957'(Harris, 1973, p. 127). Dans la pièce, le bonimenteur vend la bière en haranguant le peuple, il fait donc double boulot; c'est une technique dramatique à laquelle Césaire a recours pour faire résumer la lutte de Lumumba avant l'indépendance tout en s'inspirant de faits réels. La didascalie informe le lecteur que le bonimenteur est sous les yeux de deux flics belges, ainsi était la censure de la colonisation, chaque comportement, chaque mot sont sous la surveillance des flics. Nous dégageons quelques mots de ses boniments:

Buvez! Buvez donc! D'ailleurs, n'est-ce pas la seule liberté qu'ils nous laissent ? On ne peut pas se réunir, sans que ça se termine en prison. Meeting, prison! Ecrire, prison! Quitter le pays ? Prison! \{...\} Mais voyez vous-mêmes! Depuis un quart d'heure, je parcours le pays, et leurs flics me laissent faire! Motif: je vends de la bière ! Si bien que l'on peut affirmer que le bock de bière est désormais le symbole de notre droit congolais et de nos libertés congolaises! (Césaire, 1973, p. 11)

Ainsi expose Césaire le vrai visage de la colonisation au Congo. La colonisation annule toutes les formes de la vie, toutes les formes de la liberté, mais elle laisse une seule liberté : la Bière. La bière, que le colonisateur apporte comme une forme de civilisation, est la seule forme de liberté. Si Césaire s'inspire du réel, il l'expose dans la pièce pour dénoncer ces mœurs. Le style est marqué par les points d'exclamation pour renforcer le ton ironique. La prison, avec toutes ses connotations négatives, est le moyen que Césaire a choisi pour résumer la répression du colonisateur.

Mokutu annonce que Lumumba est tombé prisonnier: 'j'ai du neuf à vous vendre. Les Flamands ont arrêté Patrice, rein à faire pour les attendrir" (Césaire, 1973, p. 16). Cette séquence se termine par la scène des geôliers se moquant de Lumumba, alors que le président de la prison 
vient pour l'informer qu'il va participer à la conférence de La Table Ronde de Bruxelles; cette conférence fixe le 30 juin comme jour de l'indépendance. Le jour de l'indépendance était un jour historique non seulement parce qu'il apporte un événement historique, mais aussi parce qu'il témoigne le discours historique de Lumumba. Trois discours sont prononcés: le discours de Basilio (le roi de Belges), celui de Kala-Lubu (le président) et celui de Lumumba ${ }^{3}$. Jeter la lumière sur le contenu de ces trois discours est très important, car ils incarnent évidemment la relation entre le colonisateur et le colonisé pendant un moment assez critique. Nous en dégageons les propos les plus significatifs: Basilio (le roi de Belges):

Le peuple barbare, jadis terrassé par la rude poigne de Boula Matari, nous avons pris en charge. Eh oui, la providence nous a commis ce soin, et nous l'avons nourri, soigné, éduqué. Si nos efforts ont pu vaincre leur nature, si nos peines rencontrent salaire, par cette indépendance qu'aujourd'hui je leur apporte, nous allons l'éprouver. (Césaire, 1973, p. 25)

Ce discours condensé comporte des mots péjoratifs. Nous commençons par l'adjectif barbare dont le contraire, dans le contexte de la pièce, est "civilisateur". Ce ton supérieur nous le trouvons dans toutes les pièces de Césaire le mot "civilisation" était toujours le prétexte pour justifier la colonisation. Puis il cite le nom de Boula Matari; l'appellation de ce nom est reprise pour désigner la bienfaisance de la Belgique à l'égard du peuple congolais. Boula Matari est un surnom donné par les Congolais à l'explorateur Henry Morton Stanley ${ }^{4}$ qui signifie le "briseur de rochers ", il était connu par sa violence contre les nègres. Certains critiques voient que le discours apporte aussi un ton paternaliste avec les verbes : prendre en charge, soigner, éduquer et nourrir (Mbom, 1979, p. 143), mais nous ne voyons dans l'emploi de ces verbes que des insultes pour humilier les Congolais. La fin de la citation désigne l'avis du roi des Belges qui traitent les Congolais comme des ingrats à cause de leur nature. Ils ne

\footnotetext{
${ }^{3}$ Aux annexes de son Livre "Quand Césaire écrit Lumumba parle”, Suzanne Houyoux ajoute une copie de ces trois discours, en lisant les trois discours réels, on trouve que Césaire donne le lecteur le contenu de ces discours mais avec ses interprétations personnelles. L'auteur de "Aimé Césaire, "Une saison au Congo: étude critique "voit que Césaire reste fidèle à la réalité de tels discours.

${ }^{4}$ Est un explorateur britannique connu par ses explorations en Afrique et aussi par son hostilité contre les nègres.
} 
peuvent pas vaincre leur mauvaise nature ${ }^{5}$ puisqu'ils luttent contre leurs bienfaiteurs; c'est pourquoi il accepte de leur donner l'indépendance.

Le discours de Kala- Lubu reflète bien son accord, son hommage et sa collaboration avec le roi des Belges. Nous en dégageons quelques mots : il commence par le mot "Sire"; il remercie le roi pour sa protection: "ces populations que vous avez aimées et protégées"; il décrit la relation entre les Belges par l'amitié: "l'amitié que la Belgique leur offre, dans la voie d'une collaboration" (Césaire, 1973, p. 27). Ces mots sont suffisants pour dévoiler la tendance du nouveau président du Congo indépendant.

Avant d'exposer le noyau du discours de Lumumba, il vaut mieux citer que Lumumba n'avait pas le droit de parler selon le protocole de ce jourlà, mais, après avoir lu les deux discours, la veille du 30 juin, son ami Van Lierde lui propose de prendre la parole pour "réagir contre ces deux discours paternalistes et stupides"6 (Houyoux, 1993, p. 84).

À travers un discours significatif, assez sensible, Lumumba réfute les deux discours précédents, la lutte, la souffrance, l'humiliation ne devraient jamais, par des compliments ridicules, être oubliées en les qualifiant par l'amitié ou bien la collaboration. C'est grâce à la lutte que les Congolais ont obtenu leur indépendance; l'indépendance n'est jamais un don, mais elle est le fruit de cinquante ans de souffrance, d'ailleurs les Congolais doivent la goûter bien.

Toute souffrance qui se pouvait souffrir, nous l'avons soufferte. Toute humiliation qui se pouvait boire, nous l'avons bue ! Mais camardes, le goût de vivre, ils n'ont pu nous l'affadir dans la bouche, et nous avons lutté, avec nos pauvres moyens, lutté pendant cinquante ans. Et voici nous avons vaincu ${ }^{7}$

\footnotetext{
${ }^{5}$ Leur nature désigne ici la malédiction de Cham, une conviction religieuse dans la Bible, cette malédiction atteste que l'homme noir est maudit. Canaan est le fils de Cham est maudit par son grand-père Noé: “Maudit soit Canaan! Qu'il soit l'esclave des esclaves de se frères". Le Figaro: https://bit.ly/34yVmUL

${ }^{6}$ Van Lierde raconte qu'il dit à Lumumba en le conseillant "le micro sera à deux mètres de toi, personne n'osera marcher sur tes pieds, si tôt que les deux orateurs ont fini, tu t'en empares". De cette manière assez comique en tant que courageuse, Lumumba prononce un discours assez significatif et assez hardi tout en terrifiant les hommes d'intérêts au Congo.

${ }^{7}$ La poétique de ces mots appartient à Césaire, mais les idées appartiennent au vrai discours de Lumumba.
} 
(Césaire, 1973, p. 28).

Le passage par ces trois discours peut suggérer comment sera la séquence de l'après indépendance : l'arrogance et la frustration de la part de Basilio après le discours de Lumumba, la collaboration de Kal-Lubu et l'imprudence de Lumumba. Chaque discours sera traduit par des actes pendant la saison de Lumumba.

Le premier jour après l'indépendance fait paraître tant de problèmes. Les soldats criant "A mort Lumumba ! Lumumba pamba" ${ }^{8 ، "}$ (Césaire, 1973, p. 34). C'était le cri demandant l'africanisation totale et immédiate des cadres ${ }^{9}$. Cette mutinerie, contre Lumumba, se termine par l'accord: “A chacun de vous donc, le gouvernement accorde la promotion aux gardes supérieurs : le soldat de première classe devient sergent, le sergent adjuvent"(Césaire, 1973, p. 36). Les soldats demandent aussi l'écartement du général Massens en choisissant Mokutu pour être le chef d'état-major, la réponse de Lumumba est la ratification: "Vous choisissez Mokutu. Soit ! J'ai ratifié votre choix. Je sais que Mokutu ne me trahira jamais"(Césaire, 1973, p. 37). Mais les caisses sont vides, et les soldats ne sont pas prêts pour la nouvelle promotion et Mokutu sera le premier à le vendre. Le style de Césaire apporte assez de critiques contre le comportement de Lumumba, il leur donne la satisfaction immédiate de tout ce qu'ils exigent, ainsi qu'il parle au nom du gouvernement en prenant des décisions importantes. Nous pouvons mesurer la critique de Césaire en lisant les propos d'un ministre disant: “c'est gai! Il commence bien l'indépendance" (Césaire, 1973, p. 35). La scène commençant par le cri: "la mort à Lumumba" est finie par un autre cri assez opposé: “Oui! Oui! Vive Lumumba" (Césaire, 1973, p. 37). Cette procédure poétique permet la critique contre les deux parts ensemble: les soldats, à cause de

\footnotetext{
${ }^{8}$ Le mot "pamba"signifie une personne vulgaire en Lingala (une langue bantoue)

${ }^{9}$ Dans son livre "l'humanisme dans le théâtre de Césaire" Harris Rodney éclaire la question de l'africanisation : selon les accords passés entre la Belgique et le Congo, l'armée avec ses soldats congolais et ses officiers Belges devaient, au lendemain de l'indépendance, passer tout entière aux ordres du gouvernement congolais. Il était entendu que peu à peu les officiers belges soient remplacés par des chefs autochtones. Cette mesure, approuvée par Lumumba fut violement refusée par les soldats qui réclament l'africanisation totale et immédiate.
} 
leur facilité et leur irresponsabilité de la part des soldats, et Lumumba ${ }^{10}$ à cause de son imprudence.

La suite de la pièce comprend des événements importants se succédant rapidement les uns après les autres. Au jour où la population blanche quitte le Congo, des actes de violence ont été commis par l'armée congolaise contre les colons belges, ce qui fournit à la Belgique la chance d'intervenir au Congo. Le refus de l'atterrissement de l'avion de Lumumba à Elisabethville annonce la sécession de Katanga ${ }^{11}$. Katanga est une région Congolaise importante, car les Belges ont transformé 80\% de l'économie congolaise à cette région. La sécession du Katanga annonce la guerre civile, nous ajoutons à ces problèmes les problèmes à l'intérieur du gouvernement de Lumumba, par exemple ceux entre Mokutu et M'Polo' 12: 'J'apprends que M'Polo se balade partout $\{\ldots\}$ Et en tout cas, qu'entre nous deux, le gouvernement choisisse ou lui, ou moi!" (Césaire, 1973, p. 56). Tous ces problèmes ne sont pas nés par hasard, mais par des complots belges, Lumumba condense ces problèmes en quelques mots: "les Belges sont encore au Congo" (Césaire, 1973, p. $61)$.

Harris Rodney voit que Césaire effectue quelques changements dans l'ordre chronologique des événements historiques dans sa pièce pour mettre en évidence le rôle des Belges : la sécession du Katanga s'est déclarée indépendante le 11 juillet, on l'apprend dans la scène 11 . Le refus de l'atterrissage s'est produit le 12 juillet, on l'apprend dans la scène 10, la rupture avec la Belgique qui est datée le 14 juillet est présentée dans la scène 9 (Harris, 1973, p. 140). Césaire présente la rupture en avance pour faire allusion au rôle des Belges dans ces

\footnotetext{
${ }^{10}$ Harris Rodney voit que cette attitude de la part de Lumumba envers les soldats est spontanée, car une armée nationale exige d'être commandé par des nationaux pas par les officiers belges.

${ }^{11}$ Katanga est la région la plus riche au Congo. Les trois personnages qui ont collaboré pour exécuter cette sécession sont: Massens (est un général que Lumumba a écarté), M'Sri et Tzumbi (sont deux dirigent d'un parti politique au Katanga.

${ }^{12}$ M'Polo est: le président de de MNC "Mouvement National Congolais" participe à la conférence de la Table Ronde reste fidèle à Lumumba jusqu'à la fin et sera assassiné avec lui.
} 
problèmes.

La guerre civile s'accroît de plus en plus: "l'A.N.C ${ }^{13}$ a eu la main lourde! Six mille Balubas ${ }^{14}$ tués! Dans l'église Saint-Jean de Bakwanga, quarante familles Balubas ont été exterminées dans des conditions incroyables de cruauté!" (Césaire, 1973, p. 66). Une grande attaque contre Lumumba, alors que le lecteur voit bien la réticence de Mokutu et de Kala-Lubu. L'attaque ne passe pas sans quelques accusations ridicules: "Lumumba a vendu le Congo aux Russes, Lumumba a vendu son âme au diable! Lumumba a reçu plusieurs millions de l'ambassadeur tchèque!" (Césaire, 1973, p. 59). La guerre civile est le prétexte que le président Kala exploite pour rejeter le gouvernement de Lumumba.

Dès les premières scènes, Césaire éclaire bien les intérêts des Belges à travers les propos des banquiers qui cherchent à faire échouer Lumumba à tout prix. Par ailleurs, il paraît bien pour le lecteur que la décolonisation est incomplète. De son côté, Lumumba demande l'intervention de l'O.N. $\mathrm{U}$, mais, sous prétexte de l'impartialité, la réponse était choquante: "l'O.N. U ne doit pas prendre parti dans un conflit intérieur" (Césaire, 1973, p. 62). Césaire ne laisse pas cette situation sans critiques: "La voilà, l'impartialité de l'O.N.U. les voilà les hommes neutres! Les armes belges et les mercenaires affluent au Congo! Il en débarque tous les jours, et vous laissez faire!" (Césaire, 1973, p. 63).

\section{Les aspects sociaux}

Comme la visée de la pièce est politique, les aspects sociaux sont peu traités de la part de Césaire. Nous ne voyons pas le peuple ou bien les couches sociales qui le constituent. Le mot "peuple" est un refrain chez tous les hommes politiques parlant au nom du peuple. À l'exception du Joueur de Sanza et Mama Makosi, il n'y a pas d'autres noms connus pour représenter le peuple; quelques rares répliques appartiennent à "un citoyen "ou à une "femme". C'est à travers ces figures que nous pouvons déceler les aspects sociaux au Congo.

\footnotetext{
13 Armée Nationale Congolaise.

${ }^{14}$ Le peuple qui vivait à Katanga.
} 


\subsection{Formes de discrimination au Congo}

Dans la pièce, le lecteur peut toucher deux sortes de discrimination : la discrimination entre le Blanc et le Noir et la discrimination entre les Noirs eux-mêmes. La discrimination entre le Blanc et le Noir paraît dans la première didascalie de la pièce: "Quartier africain de Léopoldville" (Césaire, 1973, p. 11). Susanne Houyoux cite que la capitale du Congo était divisée, avant l'indépendance, en cité européenne et cité indigène, elle note que cette ségrégation n'est pas d'origine coloniale récente mais remonte aux débuts même de l'installation des Européens en Afrique (Houyoux, 1993, p. 40). Césaire fait consciemment allusion à cette ségrégation puisqu'il consacre quelques scènes pour parler du Congo avant l'indépendance. La seconde discrimination entre les noirs est évoquée au lendemain de l'indépendance entre deux citoyens dont l'origine est différente. Césaire évoque cette querelle ethnique, au lendemain de l'indépendance, comme un problème parmi tant d'autres qu'affronte Lumumba pendant la construction d'un nouvel État indépendant. L'évocation d'une telle discrimination jette la lumière sur un grave problème social.

- Un Mukongo ${ }^{15}$ : Il faudra que tous les Bengalas ${ }^{16}$ rentrent dans leurs villages. Le pays est gâté avec tous ces Bengalas-là

- Un Mungala : C'est nous qui sommes bien des bons de tolérer qu'un Mukong soit président de la République, qu'un Mukongo nous gouverne. (Césaire, 1973, p. 24)

Quoique Césaire n'évoque rien sur les couches sociales au Congo, nous pouvons repérer l'écart dans la société congolaise. Cet écart s'aperçoit de la Revendication. La Revendication est une réplique anonyme à laquelle le lecteur lit les souhaits du peuple après l'Indépendance. La voix est évoquée de la part de Césaire pour représenter les hommes qui veulent entraver Lumumba. Pourtant nous découvrons dans cette voix, la critique des mœurs sociales qui demandent tout et tout de suite sans donner la chance au gouvernement national:

La Revendication : L’indépendance ne doit pas être un mot-vide croyez-moi,

\footnotetext{
${ }^{15}$ Mukongo sont : des groupes qui viennent de l'Afrique centrale. Le pluriel est Bakongo.

${ }^{16}$ Les Bengalas sont : des groupes ethniques viennent de l'Afrique du sud: Soudan, Camerone et Comores .le singulier est Mungala.
} 
le mot n'est pas vide $\{\ldots\}$ demandez-le à vos parlementaires et à vos ministres. Les voitures c'est pour les ministres et les députés. Les femmes ${ }^{17}$ c'est pour les députés et les ministres. Le père Noël, c'est pour les nègres à monocles ${ }^{18}$. Que le père Noël soit pour tous ! Voilà l'indépendance du Congo ! (Césaire, 1973, p. 33)

La voix de la revendication vient simultanée à la mutinerie des soldats; c'est pourquoi Césaire critique ces mœurs: “Quand je pense que pendant cinquante ans, ils ont rampé devant les Belges, et nous n'avons pas plutôt posé notre cul sur un fauteuil, que voici à nous mordre les jarrets"(Césaire, 1973, p. 37). Le style évoque ici l'opposition du comportement du peuple. Mais nous constatons que cette voix évoque une vérité sociale: il y avait un fossé entre les politiciens et le peuple. Une didascalie au début de la scène huit du deuxième acte désigne "l'appartement de Lumumba" alors qu'une autre didascalie au début de la scène onze du deuxième acte désigne "villa de Lumumba" ce qui montre que Lumumba a un appartement et une villa.

\subsection{Statut de la femme au Congo}

Nous revenons aux deux figures anonymes le Mama Makosi et le joueur de Sanza. La figure de Mama Makosi rappelle le statut de la femme au Congo. Dans la pièce, nous distinguons trois figures pour la femme congolaise, nous les présentons par l'ordre d'entrée en scène.

\subsubsection{La femme libre}

La première figure paraît dans la didascalie de la deuxième scène de la première partie est celle des "femmes libres" (Césaire, 1973, p. 14). Du premier coup, l'épithète libre ajoutée au nom "la femme" nous donne une connotation positive ${ }^{19}$, mais Susanne Houyoux cite que les femmes libresz connotation positive ${ }^{20}$, mais Susanne Houyoux cite que les

\footnotetext{
${ }^{17}$ Ces propos évoquent comment était la position de la femme dans la société congolaise.

${ }^{18}$ Susanne Houyoux cite qu'au Congo, on dit un nègre à monocle pour désigner que cette personne est impérialiste.

${ }^{19} \mathrm{Il}$ s'agit de la différence à la conception culturelle, chez nous, les Arabes, quand on dit une femme libre : ça donne le sens d'une femme vertueuse; c'est le contraire d'une femme esclave.

${ }^{20}$ Il s'agit de la différence à la conception culturelle, chez nous, les Arabes, quand on dit une femme libre : ça donne le sens d'une femme vertueuse; c'est le contraire d'une femme esclave.
} 
femmes libres font allusion au problème de la prostitution au Congo; il était d'usage qu'une des filles de la famille ne soit pas donnée en mariage pour pratiquer la prostitution afin d'assurer le confort matériel de ses parents (Houyoux, 1993, p. 52). Les notes d'Houyoux assurent que la société congolaise rend la femme un produit à vendre. Césaire jette la lumière sur ce problème social tel qu'il est sans le critiquer; il se contente de faire allusion à ce problème à travers une scène dans un bar.

\subsubsection{La femme patriote}

La seconde figure de la femme est celle de Mama Makosi, Houyoux note que "Mama Makosi" a une existence référentielle. Il s'agit d'une femme corpulente à la langue bien pendue qui servait d'agent publicitaire à la Polar. Elle ajoute, appeler ainsi une femme c'est une marque de respect (Houyoux, 1993, p. 72). Mama Makosi représente une autre catégorie de la femme congolaise; elle est sage, puissante et patriote. Elle est un personnage collectif du peuple; c'est pourquoi Lumumba la respecte et l'apprécie. Elle peut réagir au temps difficile; quand Lumumba est arrêté par les Belges, le peuple est divisé entre : faire grève ou rester en deuil, mais elle a un autre avis assez sage: "Ni deuil, ni grève. Le travail, c'est le travail. On travaillera plus que jamais. On paiera une caution aux Belges" (Césaire, 1973, p. 17). Quand Lumumba échappe aux contrôles de l'A.N.C, il se réfugie au bar de Mama Makosi, il prend de ce bar un lieu officiel pour rencontrer les journalistes et les politiciens ${ }^{21}$. Mama Makosi reste fidèle à Lumumba même après son assassinat, au temps que la foule crie "Vive Mokutu! Mokutu Uhuru!" elle est la seule criant "Uhuru Lumumba" (Césaire, 1973, p. 115).

\subsubsection{La femme-épouse}

La troisième figure est celle de Pauline, la femme de Lumumba, ${ }^{22}$ cette

\footnotetext{
${ }^{21}$ Houyoux note qu'à la vérité quand Lumumba échappe aux contrôles de l'A.N.C, grâce à quelques soldats, il fait une brève escalade et se rend dans l'un de ses bars favoris, mais il refuse de quitter sa cellule jusqu'au jour de son transfert au Katanga, puis son assassinat. Césaire s'inspire cet ajout de la réalité pour montrer que Lumumba est très attaché au peuple (Houyoux, 1993, p. 209)

${ }^{22} \mathrm{C}$ 'est la deuxième femme de Lumumba, avec elle, il a quatre enfants, après assassinat de Lumumba, elle s'évade en Égypte. C'est en Égypte qu'elle poursuit toute seule l'éducation de ses enfants avant d'être ramené au Congo en 1967.
} 
figure représente la femme mère et épouse qui est assez inquiète pour son mari. Elle paraît sage et intelligente, c'est elle seule qui avertit Lumumba de la trahison de ses compagnons :

Pauline: Patrice, j'ai peur $\{\ldots\}$ Je vois partout des termites, des crapauds, des araignées $\{\ldots\}$ je crois se resserrer autour de toi...

Lumumba: Pourquoi avoir peur ? C'est vrai j'ai des ennemis, mais le peuple est avec moi.

Pauline: Le peuple oui, mais le peuple est faible et désarmé! Et tes ennemis sont puissants!

Lumumba: Il ne faut pas exagérer .... J'ai des amis aussi ! Et des amis fidèles!

Pauline: Certains te vendraient pour un plat de lentilles!

Lumumba: Oh ! Les femmes ! Peuvent-elles être mauvaises! Toujours à imaginer le pire!". (Césaire, 1973, p. 72)

Nous commençons par le style qui comprend tant de signes culturels; les métaphores de Pauline décèlent bien la société empoignante. Ces bêtes vilaines et ces insectes reflètent l'environnement congolais. Le choix de ces bêtes est pertinent, car ces bêtes nuisent à la société. L'expression proverbiale "vendre pour un plat de lentille" s'accorde bien avec la jalousie de Kala et la malice de Mokutu. Le dialogue entre Pauline et Lumumba indique que Pauline est prévoyante, elle n'imagine pas le pire, car elle a ses preuves : Kala est jalouse de Lumumba et Mokutu est espion des Belges; mais Lumumba en est très confiant. Quand les faits s'intensifient, Pauline ne peut pas cacher ses sentiments en telle qu'elle est épouse et mère dont la vie de son mari est menacée: "Tu es toujours têtu, intraitable, une tête de fer, $\{\ldots\}$ Veux-tu qu'en jour, la tête rasée, à suivre un cortège funéraire ? Et les enfants, en feras-tu des orphelins?" (Césaire, 1973, p. 95).

Nous jetons la lumière sur le statut de la femme, car cela décèle des côtés marginaux dans la pièce. La femme libre fait émerger le problème de la prostitution au Congo, la figure de Mama Makosi trace un portrait positif de la femme patriote, alors que Pauline Lumumba est le symbole de la femme fidèle, prévoyante.

Clément Mbom voit que le rôle de la femme dans le théâtre de Césaire est négatif ou absent; il voit qu'elle y est quasi absente dans "Une Tempête"et son rôle est, car elle (Pauline) est "démobilisatrice du 
révolutionnaire". En ce qui concerne Mama Makosi, il voit que c'est sa sympathie pour Lumumba qui lui permet de réagir dans certaines circonstances: "les femmes qui paraissent dans le théâtre de Césaire occupent une place marginale" (Mbom, 1979, p. 98). Il pose la question suivante : "peut-on parler d'une certaine misogynie ${ }^{23}$ dans le théâtre de Césaire ?" (Mbom, 1979, p. 98). Il voit que le regard de Césaire pour la femme est un regard négatif. Selon ce regard, la femme est une détentrice de la production et de la reproduction. Autrement dit, elle produit des enfants (c'est la production) et les conserve pour continuer la société; c'est pourquoi il est difficile d'être révolutionnaire, car la révolution menace sa production (Mbom, 1979). Ainsi, justifie Mbom le regard négatif de Césaire pour la femme. Pour plus d'une raison, nous ne nous accordons pas avec cet avis: en premier lieu, le rôle de la femme dans le théâtre de Césaire n'est pas négatif, il est vrai que son rôle est marginal mais il n'est pas négatif. Pauline n'est pas démoralisatrice mais prudente. La réaction de Mama Makosi désigne bien qu'elle était raisonnable, sage et patriote. En second lieu, en lisant "Aimé Césaire", un livre détaillé sur la vie et l'œuvre de Césaire, de Reaulaud Fankoua, nous découvrons que le regard de Césaire est respectueux envers la femme. Césaire parle de sa grand-mère et de sa professeure avec une grande considération. Susanne, la femme de Césaire, détient un rôle illuminé dans la société martiniquaise et dans la revue de La Présence Africaine. Par ailleurs, le regard de Césaire envers la femme n'est pas rétrograde, comme le dit $\mathrm{M}$. Mbmo, mais c'est le cadre de la pièce qui limite le rôle de la femme. En tant que pièce politique engagée, le sujet traité marginalise tous les aspects sociaux parmi eux la femme et son rôle.

\subsection{Le peuple et la maturité politique}

Nous reprochons à Césaire l'exagération dans quelques répliques concernant la maturité des Congolais. Dans les rares répliques qui appartiennent au peuple, le lecteur entend un citoyen dire : "C'est quoi au juste, votre Dipenda ${ }^{24}$ ?” (Césaire, 1973, p. 23). L'autre se demande:

\footnotetext{
${ }^{23}$ Aversion pour les femmes.

${ }^{24}$ Houyoux note que c'est la déformation Congolaise de l'indépendance (Houyoux, 1993, p. 67)
} 
"Comment elle arrive, Dipenda? En auto, en bateau, en avion ?" (Césaire, 1973, p. 23). L'Histoire du Congo nous apprend qu'il y avait beaucoup d'émeutes et de révoltes avant l'indépendance, par ailleurs, comment faire passer de tels questionnaires ridicules. Harris Rodney justifie cette exagération en disant que Césaire veut montrer "le manque de la maturité de peuple" (Harris, 1973, p. 133), il le pose comme l'un des problèmes qu'affrontent Lumumba en particulier. Il est vrai que, chaque nouvel État indépendant a connu ce manque de la maturité ce problème, mais nous voyons que c'est une exagération ridicule de la part de Césaire.

Puisque nous cherchons à mesurer l'évolution dans la société congolaise, nous devons noter que tous les lieux populaires cités dans la pièce sont des bars, la consommation de la bière est très répandue et partout chez les politiciens ainsi que chez le peuple. Houyoux affirme que la boisson alcoolisée est autorisée au Congo. Lumumba avait également travaillé comme directeur commercial dans une brasserie (Houyoux, 1993) mais cette coutume est intruse, le colonisateur l'apporte comme un signe civilisateur.

\subsection{Le joueur de Sanza ${ }^{25}$}

Le joueur de Sanza est une figure anonyme. C'est la figure qui, selon Césaire, représente le peuple. Dans la première version, le joueur de Sanza est une sorte d'intermède; il commence et conclut la scène. Dans la seconde version, Césaire enrichit le rôle du joueur de Sanza, Il commente cette modification en disant:

Pour moi, il incarne le peuple: c'est l'esprit populaire, l'esprit de bon sens qui servit à toutes les occupations. Ce n'est pas parmi les élites qui sont occidentalisées, ni chez les puissants qu'on le trouve. Il est là, il survit, cet esprit, au niveau de Folklore, au niveau du petit peuple, et c'est le Congo que représente le joueur de Sanza. (Harris, 1973, p. 125)

Quand le joueur de Sanza parle, nous lui donnons de l'importance: ses paroles soit en prose soit en vers, sont symboliques, significatives et énigmatiques. À sa voix, nous entendons la voix du peuple. La richesse

\footnotetext{
${ }^{25}$ Le joueur de Sanza ressemble beaucoup شاعر الريابة en Égypte. Le Sanza est un instrument de musique, Houyoux n'identifie pas ce personnage comme un témoin des événements, mais elle voit qu'il n'est pas d'une pure fiction.
} 
de ses mots peint des images pleines d'énigmes et de symboles à travers lesquelles le lecteur peut découvrir la culture, et l'environnement. À la fin de la pièce, la dernière didascalie informe le lecteur que le joueur de Sanza tombe victime de la garde de Mokutu. Son rôle sera exposé en détail aux aspects culturels en tant que figure folklorique.

\section{Les aspects culturels}

La culture, dans le théâtre de Césaire, devient un instrument important dans la lutte politique. Si Césaire marginalise les côtés sociaux, il enrichit les aspects politiques. Nous constatons que c'est une réaction psychologique de la part d'un exporté privé de sa langue, sa religion et son folklore. Quand Césaire est allé à Paris, il n'avait pas de nostalgie pour la Martinique: “je n'avais pas de nostalgie du tout, ce qui fait que j'étais ouvert sur le monde nouveau où j'allais" (Césaire, 2007, p. 28). Mais quand il découvre L'Afrique, grâce à Senghor ${ }^{26}$, cela évoque sa nostalgie pour L'Afrique : "Le jour même de mon inscription au lycée Louis- le- Grand, je découvre un autre monde, c'est l'Afrique (...) mon africanité inconsciente se révélait quand Senghor m'expliquait les choses"(Césaire, 2007, pp. 28,29).

L'évocation de la culture africaine vient comme une réaction contre la privation. Dans la présente pièce, le lecteur peut découvrir tant d'aspects culturels: chants, danse, proverbes, contes, termes et expression bantous ${ }^{27}$

\subsection{La langue}

La langue régionale au Congo est le swahili. "Uhuru" en swahili veut dire la liberté, nous le lisons plus d'une fois. Les temps auxquels l'auteur emploie les langues africaines sont bien significatifs: avant l'indépendance, au cours de la guerre civile et aux moments difficiles. La langue africaine paraît comme un abri. C'est une allusion de la part de Césaire pour dire que c'est au temps difficile qu'on se réfugie à notre

\footnotetext{
${ }^{26}$ C'est Senghor qui l'a fait découvert Leo Frobenius dont l'œuvre est intitulée:

"Histoire de la littérature négro-africaine" à travers lequel, Césaire découvre la culture et la civilisation africaines.

${ }^{27}$ On nomme Bantous (les "Humains “en kongo) les locuteurs d'un ensemble de langues dites bantoues qui regroupe environ quatre cent cinquante langues sur le continent africain.
} 
langue originale, comme si le recours à notre langue nous donne la force et la stabilité.

Nous lisons quelques mots, à la fin de la scène trois du premier acte: "Kongo Mpaka Dima” (Césaire, 1973, p. 20). Houyoux décode ces mots: Kongo est le nom de l'ancien royaume africain qui comprenait notamment l'aire territoriale de l'actuelle République du Zaïre. En swahili Mpaka signifie "jusqu'à" et Dima veut dire "toujours", par ailleurs l'expression peut traduire "Le Congo pour l'éternité" (Houyoux, 1993, p. 60)

Avant la guerre civile, c'est aussi le joueur de sanza qui crie en annonçant la guerre par le terme suivant: "Luma! Luma!"(Césaire, 1973, p. 40). Luma est un terme qu'on peut trouver dans plusieurs langues bantoues, en lingala par exemple luma signifie faire souffrir; de façon générale le terme équivaut à : piquer, blesser, faire mal, frapper ou encore copuler (Houyoux, 1993).

\subsection{Proverbes et maximes}

La lecture de la présente pièce décèle une autre partie de la culture africaine celle des proverbes et des maximes africains. Nous commençons par cette maxime: "Nous sommes comme les poils du chien. Tous couchés sur la même couchette”(Césaire, 1973, p. 72). Houyoux commente que cette expression est utilisée par les anciens pour clore le palabre (Houyoux, 1993). L'expression incite à l'unité puisqu'ils ont le même sort.

En justifiant sa confiance en Kala, Lumumba cite la maxime suivante: "lorsqu'on voit le bec du coq, on voit le coq tout entier!" (Césaire, 1973, p. 73) Kala est le chef d'une ethnie puissante. Houyoux note que cette maxime est inspirée du culte des anciens ${ }^{28}$ (Houyoux, 1993).

Ghana, un soldat appartient aux forces nationales de l'O.N.U., quand il refuse de laisser Lumumba parler à la radio, il cite le proverbe suivant: "L'État est un œuf, trop serré il se casse! Pas assez, il tombe et se brise"

\footnotetext{
${ }^{28}$ Elle cite le texte des anciens : Faites que dans les savanes que nous habitons. Il y ait bonne entente parmi nous. Que lorsqu'on voit le bec du coq. On voit le coq tout entier. La maxime ressemble beaucoup le sens qu'apporte le proverbe suivant: "A l'ongle on connait le lion".
} 
(Césaire, 1973, p. 77). Ce proverbe veut dire que la personne au pouvoir ne doit être ni tyran ni lâche. Le proverbe est inspiré d'une chanson connue dont les mots sont les suivants: 'L'amour est comme un œuf. Qu'il faut tenir fortement. S'il tombe il se casse et il ne reste que les regrets" (Houyoux, 1993, p. 178).

Le proverbe suivant est dit de la part d'Okto à Lumumba: "Nous mangeons avec le soleil, nous ne mangeons pas avec la lune"(Césaire, 1973, p. 86).Avant de lire le livre d'Houyoux, nous voyons que ce proverbe incite à être rational pour résoudre les problèmes loin de rêves $^{29}$, surtout la scène du proverbe montre que Lumumba raconte un rêve à Okto. Le soleil désigne le jour où les hommes travaillent, alors que la lune désigne la nuit où les hommes dorment. Mais Houyoux donne une autre interprétation à ce proverbe; elle voit que l'Homme vit et prospère sous le soleil, mais les esprits malfaisants vivent sous la lune:

Le soleil et la lune deux séries d'éléments antagonistes : jour et nuit, lumière et ténèbres, monde des hommes et monde des esprits, mâles et femelle, ordre et désordre. Les pratiques magiques associées à la lune obscure se reportent à ce mode de classification et d'interprétation de l'univers (Houyoux, 1993, p. 194)

Parmi toutes les expressions qui concernent la société congolaise, une expression prononcée par le geôlier: "on ne fera pas du matata"(Césaire, 1973, p. 88). Faire matata veut dire : faire des histoires. Houyoux note que le mot "matata" signifie: "des difficultés, des ennuis, des querelles; l'expression est toujours utilisée au pluriel” (Houyoux, 1993, p. 196).

\subsection{Les chants}

Le joueur de Sanza $^{30}$ est une figure importante. Il paraît au début de la pièce en chantant sous les yeux des flics qui le comparent à une mouche: "c'est le joueur de sanza, pas dangereux $\{\ldots\}$ une vraie mouche, et toujours à bourdonner"(Césaire, 1973, p. 13). Césaire fait voir comment le colonisateur mesure la culture du colonisé; le joueur de sanza est mesuré sur la balance de sécurité: est-il dangereux ou non? Ce n'est pas une question de culture pour eux. L'extrait comprend aussi un sens sousentendu: c'est le colonisateur qui a toujours peur. La métaphore de la

\footnotetext{
"الأحلام لا تجلب قوتًا" :

${ }^{30}$ Le Sanza est instrument de la musique au Congo. Voir l'annexe.
} 
"mouche" exprime l'ironie de Césaire, c'est l'incompréhension des flics qui mesure le danger du joueur de Sanza; ils le critiquent sans comprendre ce qu'il chante.

L'apparition du joueur de sanza est toujours significative comme ses mots. Il chante, après l'arrestation de Lumumba de la part de la Belgique. Il critique la colonisation tout en donnant l'espoir à la décolonisation:

Vienne le temps des pluies,

Viendra aussi la guerre,

Le temps du Sang rouge

Est le temps que j'annonce,

Puissant est le buffle, et puissant l'éléphant, Bientôt tombera le buffle. Et bientôt l'éléphant

Le temps que j'annonce est le temps du Sang rouge.

La liberté est pour demain (Césaire, 1973, pp. 17-18).

Le subjonctif exprime le désir, la pluie exprime l'espoir, car la pluie est toujours le symbole de la vie et du bonheur. Le buffle et l'éléphant sont des métaphores qui comparent les Belges et les flamands ${ }^{31}$ aux deux animaux.

Après le discours de Lumumba et les réactions qu'il incite, le chant suivant était une alerte pour Lumumba, le joueur de Sanza prévoit les compromis de ses compagnons qui aiment l'argent et le pouvoir, ces hommes sont assez hypocrites, ils savent bien cacher leur mauvaise intention:

À prendre le vent,

Nul n'est plus qu'eux habile

Ils n'ont pas gueules d'assassins

Mais blair à flairer toute odeur dans le vent

Ce sont hommes d'appétit

Pour la bouffe tout leur est râtelier. (Césaire, 1973, p. 31)

Le joueur de sanza passe toujours avant ou après les répliques en apportant les interprétations personnelles de Césaire:

Malafoutier ${ }^{32}$ qui monte jusqu'au haut du palmier,

\footnotetext{
${ }^{31}$ Les Flamands sont les habitants de la Flandre ou ceux qui - habitant d'autres régions parlent le néerlandais ou des dialectes proches en Belgique.

${ }^{32}$ Le nom Malafoutier est créé du terme malafu. Le Malafoutier est la personne dont l'occupation principale est de récolter la sève des palmiers consommés comme vin de palme.
} 
Descends, petite fourmi,

Descends petit passereau

Les bonnes âmes chantent au pied du palmier

Malafoutier tu montes, tu montes

Passereau de liberté ivre! (Césaire, 1973, p. 42)

La Malafoutier renvoie à Lumumba, la petite fourmi et le petit passereau renvoient à ceux qui l'entravent. L'adjectif "petite" apporte une ironie contre ceux qui veulent détruire la popularité de Lumumba. L'escalade de la palme désigne la position de Lumumba chez le peuple. Les chants du joueur de Sanza prennent parfois la forme de contes animaliers. Ses chants apportent parfois des avertissements. Les mots suivants prévoient ce qui se passera à l'insu de Lumumba. Du premier coup, c'est la musicalité de l'assonance due à la répétition du son $\{$ ã $\}$ qui attire l'attention:

Le soleil et pluie

pluie battant

soleil levant

un éléphant

fait un enfant. (Césaire, 1973, p. 61)

\subsection{La musique}

La musique et la danse sont parmi les aspects culturels évoqués dans la pièce. En fêtant l'indépendance: "on entend le cha-cha de l'indépendance"(Césaire, 1973, p. 23). Le cha-cha est "une chanson créée par Tabu Ley dit Rochereau au moment de l'accession du Congo" (Houyoux, 1993, p. 67).

Le lecteur peut connaître comment annoncer la guerre au Congo: "Le tam-tams de guerre résonnent longuement dans la nuit, transmettant la nouvelle de l'agression belge"'(Césaire, 1973, p. 40). Le tam-tam est un instrument de percussion ressemblant beaucoup au tambour chez les Arabes, il donne de la musique militaire.

\section{Conclusion}

Selon la sociocritique, il faut savoir est-ce que l'auteur a connu la société décrite ou non ? Césaire ne visite jamais le Congo, mais il puise de l'Histoire aussi bien que de sa société en leur donnant ces aspects et cette 
socialité. Césaire est, avant tout, un petit-fils d'un esclave et un fils d'un colonisé, son inconscient social puise de son expérience réelle et la transforme en faits dramatiques littéraires en décrivant la société congolaise témoignant presque les mêmes conditions.

Une saison au Congo est tranchée du réel: les faits, les personnages même les lieux sont réels. Le recours à l'Histoire pour revaloriser l'histoire est une tendance assez connue chez les dramaturges nègres (Chevalier, 2004). Ceux-ci font appel aux sociétés et aux personnages du passé pour en attirer les profits et les leçons. Césaire a habilement concilié la littérature et la réalité d'où vient la socialité que nous cherchons.

Césaire ancre le réel et le fictif dans un système de significations. Le recours à l'approche sociocritique s'explique par le fait d'analyser ce système afin d'en dégager la socialité. Tout au long de cette étude nous avons essayé de trouver et d'analyser cette socialité. Elle trouve sa place dans le déploiement et l'inscription du social dans ces textes.

L'exposé des aspects politiques, sociaux et culturels décèle bien l'image de la société congolaise témoignant l'évolution au cours des événements politiques et sociaux. La socialité exposée montre qu'au Congo l'indépendance est inachevée. La relation entre le colonisateur et colonisé reste tendue après l'indépendance. Le colonisateur voit que le Noir est incapable d'être dirigeant.

Les mœurs politico-sociales critiquées de la part de Césaire sont: la sécession, la trahison, les grandes espérances du héros face à la facilité du peuple qui veut tout et tout de suite. Les erreurs, qui entrainent l'échec du héros à la construction d'un État national, sont bien éclairées. Césaire souligne que la confiance absolue et l'intransigeance sont les grandes erreurs de Lumumba.

\section{Bibliographie}

Amossy, R. b. (2006). Analyse du discours et sociocritique. Larousse Armand Colin. Bergez, D. B. (1996). Introduction aux méthodes critiques pour l'analyse littéraire. Dunod.

Brichaux-Houyoux, S., \& Césaire, A. (1993). Quand Césaire écrit, Lumumba parle : 
édition commentée d'Une saison au Congo. Editions L'Harmattan.

Césaire, A. (1973). Une saison au Congo. Seuil.

Césaire, A., \& Louis, P. (2007). Conversation avec Aimé Césaire (Vol. 114). Arléa.

Chevalier, J. (2004). La littérature nègre. Armand, Colin.

Couprie, A. (1995). Le théâtre, texte, dramaturgie, histoire. Nathan.

Diakhate, O. (2009). Le theatre d'Aime Cesaire: Un manifeste de la dissidence.

Ethiopique uméro spécial: Hommage à Césaire.

Duchet, C. (1979). Sociocritique. Nathan.

Duchet, C. (1983, août). Entretien sur la sociocritique. (P. MAURUS, Intervieweur)

Duchet, C. (1992). La politique du texte: Enjeux sociocritiques. Presse Universitaire de Lille.

Duchet, C. (2011). Un cheminement vagabond. Honoré Champion.

Fonkoua, R. (2010). Aimé Césaire. Perrin.

Harris, R. (1973). L'Humanisme dans le théâtre de Césaire. Canada Ottawa: édition Naaman.

Galvez, M. (2014). Bibliographie de la littérature française (XVI e -XXI e siècles). Année 2013. Revue d'Histoire littéraire de la France, 11-761.

Henone, R. (1999). Aimé Césaire, le Chant blesse biologique et poétique. Jean Michel Place.

Kesteloot, L. (1999). Négritude et créolité. Francophonie et identités culturelles, 39-48.

Leenhardt, J. (2004). Sociologie de la littérature. Encyclopaedia Universalis.

Maurus, P. (2013). L'actualité de la sociocritique. Harmattan.

Mbom, C. (1979). Le théâtre d'Aimé Césaire ou la primauté de l'universalité humaine. Fenixx.

Parunier, M. (2001). L'analyse du texte de théâtre. Nathan.

Pinguilly, Y., \& Gourrat, C. (2008). Aimé Césaire, le nègre indélébile. Oskar jeunesse.

Popovic, P. (Décembre 2011). La sociocritique: Définition, histoire, concepts, voies d'avenir. Pratiques $\mathrm{N}^{\circ} 151 / 152$.

Ryngaert, J.-P. (2006). Le personnage théâtral contemporain: Décomposition et recomposition. Montreuil-sous-Bois (Seine-Saint-Denis): édition théâtrale.

Verges, F. (2005). Aimé Césaire: Nègre, Je suis nègre et je resterai: Un entretie. Albert Michel.

Zima, P. (2000). Manuel de sociocritiques. Harmattan.

Zima, P. V. (2011). Texte et société: perspectives sociocritiques. Texte et société, 1-220. 